\title{
Dynamic Virtual Network Function Placement over a Software-Defined Optical Network
}

\author{
${ }^{1}$ Sebastian Troia, ${ }^{2}$ Alessio Giorgetti, ${ }^{2}$ Andrea Sgambelluri, ${ }^{1}$ Guido Maier \\ (1) Dipartimento di Elettronica, Informazione e Bioingegneria, Politecnico di Milano, Milan 20133, Italy \\ (2) Scuola Superiore Sant'Anna/CNIT, Pisa, Italy
}

\begin{abstract}
We demonstrate how to dynamically place Virtual Network Functions over a software defined optical network integrating IT computing and real IP over WDM resources, thus allowing exchange of real traffic.

OCIS codes: (060.4265) Networks, wavelength routing; (060.4264) Networks, wavelength assignment
\end{abstract}

\section{Overview}

The growth of internet traffic in telecommunications networks is increasing dramatically. Every day, around 18 billion Internet-connected devices and 3 billion global Internet users transfer 900 Petabytes of Internet Protocol (IP) data across the globe. According to the forecasts reported by [1], global IP traffic in 2021 will be approximately three times that measured in 2016, reaching 278 EB per month.

This situation is forcing the underlying network technologies to change, increasing the level of programmability, control, and flexibility of configuration, while reducing the overall costs related to network operations. By leveraging virtualization technologies, ETSI Industry Specification Group proposed Network Functions Virtualization (NFV) to virtualize network services previously performed by dedicated proprietary hardware. NFV supplies flexible provisioning of software-based network functionalities on top of a shared physical infrastructure. It addresses the problems of operational costs of managing and controlling these closed and proprietary appliances by leveraging low cost commodity servers. Furthermore, with the advent of Software Defined Networking (SDN), the integration of SDN with NFV has seen a remarkable growth to achieve various network control and management goals. From one side, SDN helps addressing the challenges of dynamic network resource management and intelligent service orchestration; from the other side, NFV enables real-time and dynamic virtual network function provisioning.

In this demo, a flexible SDN/NFV orchestrator is used to create and instantiate virtual network functions and to connect them using a SDN-based multi-layer network, including real optical devices. The demonstration is a collaboration between Politecnico di Milano, Scuola Superiore Sant'Anna and CNIT of Pisa, within the context of the Metro-Haul project. The experimental service-orchestrator, named SENATUS [2], developed by the research group of Politecnico di Milano, implements functions for managing SDN networks as well as for deploying network services on NFV infrastructure. SENATUS targets the research environment, and it is designed to support testbeds for the development and validation of network services and network planning algorithms. As shown in Fig. 1, we use ONOS [3] as an SDN controller to dynamically manage connectivity between two endpoints in a multi-layer network, and Openstack [4] as software to manage virtual network functions. Whenever SENATUS receives a VNF instantiation request, it interacts with Openstack to manage the installation of the VNFs and at the same time queries ONOS to deploy the required connectivity on the multi-layer network infrastructure. From the SDN control point of view, with respect to previous works [5] that was totally focused on the control of the optical network, this demo integrates the orchestration tools, the control of both packet and optical devices, and allows therefore the exchange of real traffic between Virtual Machines (VMs). The network infrastructure exploited as data-plane of the testbed, as well as the SDN controllers, are provided by Scuola Superiore Sant'Anna and CNIT.

\section{Innovation}

The main innovation of this demo is the implementation of virtual network functions in a real IP-over-WDM software-defined network. For the first time, SENATUS has been exploited to integrate both IT computing and real WDM optical network resources. We deploy a typical video on demand use case in which we instantiate two VNFs: a web server for video content delivery (i.e., VNF 1) and a web client (i.e., VNF 2) that requests the video content. As depicted in Fig. 1, when SENATUS receives a request from an external entity, such as an Operations Support System (OSS), it will perform the following actions:

1- Instantiation of the VNFs through the Openstack management network

2- Computation of the shortest path (red and green lines in fig. 1) and check of the resources availability

3- Instantiation of the optical connections in the OpenROADMs at the optical layer of the network

4- Installation of the flow rules in the OpenVSwitches at the packet-switched layer 


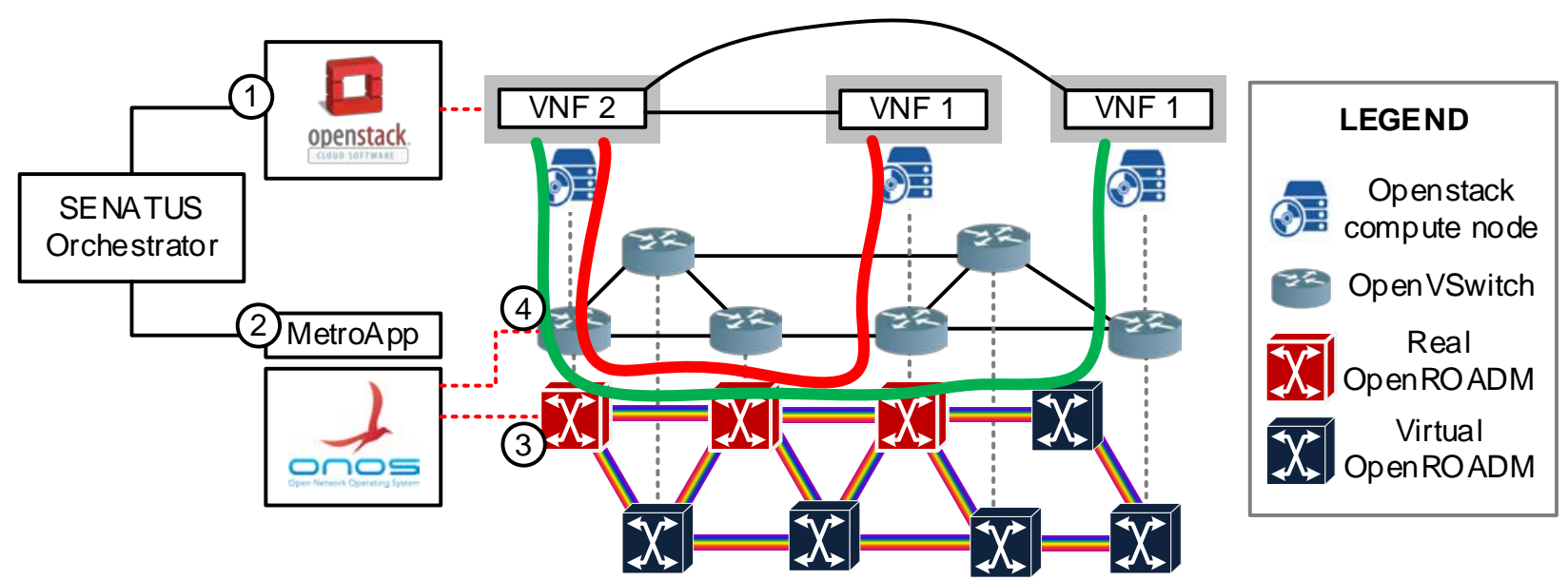

Fig. 1 SDN-NFV proposed architecture

SENATUS uses the Rest APIs made available by the MetroAPP application developed in ONOS. The ONOS controller then manages the low-level resources for setting-up the connectivity in the optical network. Once the request has been allocated, the video content can start to be delivered to the end host. The goal of the use case is to show two fundamental aspects of the demo: first, show in real-time the deployment of VNFs upon request; second, show the dynamic set up of lightpaths in the IP over WDM optical network, including the configuration of the client-side of the optical transponders that will be attached to traditional OpenFlow switches.

The demo will include the software and hardware components illustrated in Fig. 1. The SENATUS orchestrator, the ONOS controller and the OpenStack controller will be installed in one or more servers at the CNIT laboratories; two or more additional computers will be used as OpenStack compute nodes for the deployment of VMs.

The SENATUS orchestrator will communicate both with the ONOS and Openstack controllers. In the first case, it will exploit the extended REST APIs provided by the MetroAPP. Specifically, these APIs will enable SENATUS to: (1) retrieve complete network state information (including currently established lightpaths with utilized paths and optical channels); (2) require the activation of a new lightpath on the optical network (including pre-determined path and spectrum assignment); (3) require the deletion of a currently established lightpath.

In the second case, it will take advantage of OpenStack APIs in order to instantiate the VNFs. A VNF is composed by a flavor, i.e. the container of the virtual machine, and the software image, i.e. the operating system and the software that provides the VNF functionality. By invoking a single function, the service orchestrator will deploy a VNF by performing the following steps: (1) select the compute node, where to perform the instantiation and create the container; (2) build the VNF image inside the container.

The ONOS controller has been extended on the southbound interface toward the network devices. For this specific demonstration, both the OpenFlow and the NETCONF protocols are used to configure SDN switches (e.g., OpenvSwitch) and optical devices, respectively. The optical network layer includes both emulated and real network devices. All devices are equipped with a NETCONF/YANG agent (i.e., built using the ConfD tool [6]) to enable the communication with the controller. At the agents associated with real devices, the NETCONF messages arriving from the controller are translated in proprietary commands to actually configure the device (e.g., transponders are configured through proprietary HTTP messages). Specifically, real devices include three ROADMs and two pairs of transponders (i.e., the three ROADMs will have some real node degrees and some emulated node degrees), while the rest of the optical layer is emulated. Connecting the real hardware as represented in Fig. 2, a real lightpath (e.g. the red line in Fig. 1) will be set-up between the deployed VMs, and real traffic will be exchanged during the demonstration through the established lightpath. The direction from ROADM 1 to ROADM 3 physically passthrough the ROADMs, while in the opposite direction the transponders are directly interconnected. The transponders will be based on commercial Ericsson SPO 1400, each ROADM degree includes a pair of commercial Wavelength Selective Switches (i.e., Finisar Waveshaper, $1 \mathrm{x} 1$ or $1 \mathrm{x} 4$ ) and a pair of optical amplifiers, while the Add/Drop module is implemented using a fiber Coupler/Splitter, respectively.

In order to demonstrate the practical benefits of dynamic VNF placement orchestrated with network resource allocation, we designed the following experiments:

(1) VNF placement: we exploit SENATUS to perform the on-the-fly and on-demand installation of two VNFs and related network connectivity; (2) VNF migration: we will then migrate VNF 1 in another compute node (see green line in Fig. 1) and consequently reconfigure the network connectivity. 


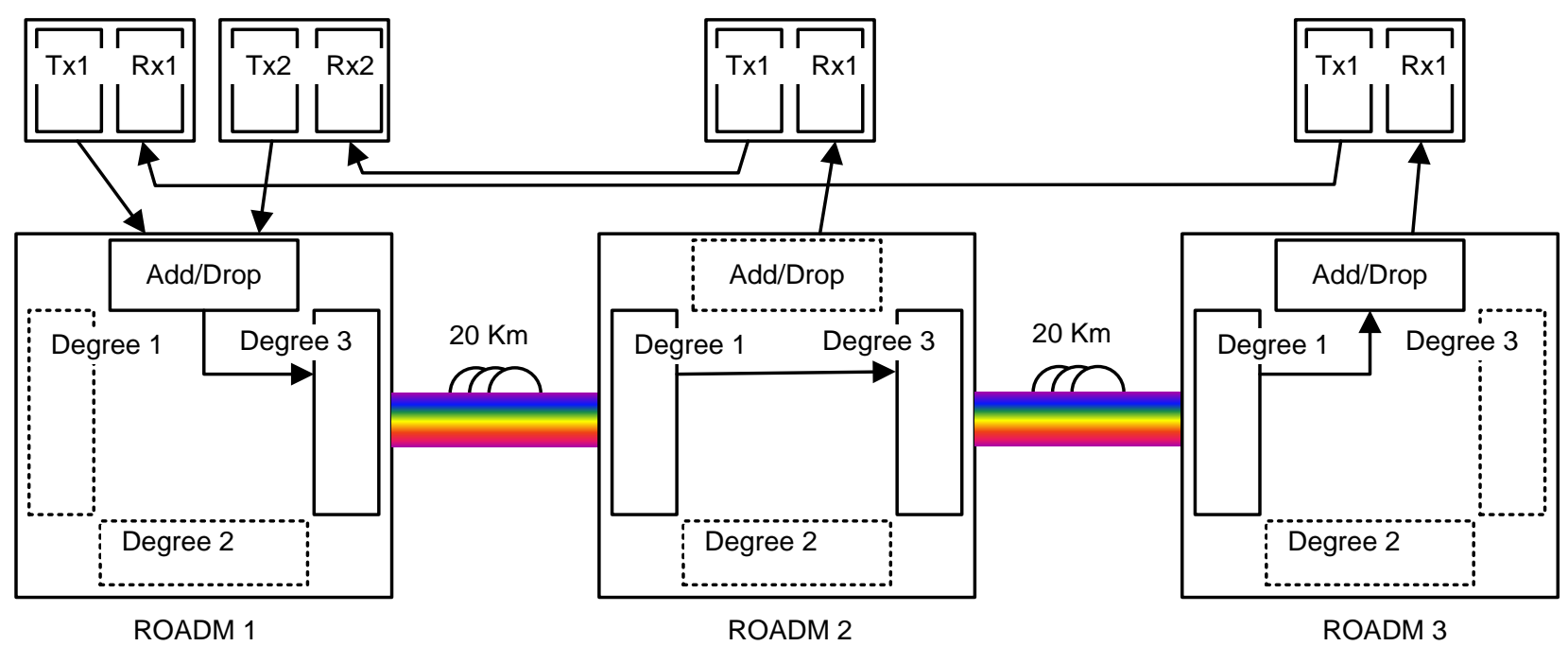

Fig. 2 The portion of the testbed including real optical devices, dotted components are emulated. Two transponders (only Tx) are physically connected to ROADM 1, one transponder (only Rx) is physically connected to to ROADM 2 and ROADM 3. ROADM 1 and ROADM 3 includes one physical degree and a physical add/drop module, ROADM 2 includes 2 physical degrees.

The testbed will be located at the CNIT premises in Pisa, including the computers hosting the SENATUS orchestrator, the ONOS controller, the OpenFlow switches and the NETCONF/YANG agents connected to the physical devices. Both the orchestrator and the controller will be therefore accessed from remote (e.g., using webbased interfaces) to trigger the demo events and to monitor the network infrastructure state (e.g., from the ONOS graphical user interface it will be possible to visualize the currently established multi-layer connections). Moreover, a pair of web-cams will be deployed at the CNIT premises. The first camera will be used to show an Optical Spectrum Analyzer (OSA) attached to one of the real optical links (i.e., showing the physical activation of the optical channel used to provide the connectivity); the second camera will display in real-time the delivery of a video flow between the two deployed VMs.

\section{OFC Relevance}

We demonstrate the use of an NFV/SDN self-developed orchestrator (SENATUS) to synchronize the deployment of computational resources and network connectivity in a multi-layer SDN network (packet over optical). We mainly use open-source tools (i.e., ONOS, OpenStack) that have been extended to test the considered functionalities. We believe that our work is of great interest for both IT service providers and telecommunication operators (optical carriers, in particular) that are more and more considering the migration of their infrastructure to the NFV/SDN paradigm.

\section{References}

[1] Cisco Visual networking Index. Forecast and methodology, 2016-2021, white paper. San Jose, CA, USA, 1, 2016.

[2] Troia, Sebastian, et al. " SENATUS: an experimental SDN/NFV Orchestrator," Proceedings of the IEEE NFV-SDN, 2018.

[3] Berde, Pankaj, et al. "ONOS: towards an open, distributed SDN OS." Proceedings of the third workshop on Hot topics in software defined networking. ACM, 2014.

[4] Sefraoui, Omar, et al. "OpenStack: toward an open-source solution for cloud computing." International Journal of Computer Applications 55.3 (2012): 38-42.

[5] A. Sgambelluri et al, "Fully Disaggregated ROADM White Box with NETCONF/YANG Control, Telemetry, and Machine Learning-based Monitoring”, in Tech. Dig. OFC 2018.

[6] Available at http://www.tail-f.com/confd-basic/, by Tail-f a Cisco Company 